\title{
Receptor Binding Techniques
}

Initial interest in radioligand autoradiography of brain tissue was driven by the potential value of this technique in studying the in vivo distribution of centrally acting drugs - e.g., anticonvulsants and sedatives - and in evaluation of their mechanisms of action and pharmacokinetics (Roth and Barlow, 1961). A variety of clinically useful agents had been discovered empirically through behavioral screening, but without clear indication as to their biochemical actions in the brain. Subsequently, it was recognized that many drugs interacted with neurotransmitter receptors or other minor protein constituents by means of stereospecific, saturable binding. The in vivo distribution of radioligands was then applied to detailed mapping of the binding sites in brain (Yamamura et al., 1974). It was later determined that in vitro assays employing radioligands could be performed for the same purpose (Young and Kuhar, 1979). The potential advantages of the in vitro design became immediately apparente.g., the ability to directly control and measure aspects of the binding reaction, the use of reduced amounts of radioligand, and the possibility of employing radioligand binding to evaluate the properties of unlabeled drugs in competition assays.

In vitro autoradiographic binding-site assays are now important tools in contemporary neuroscience and pharmacology, with strengths and limitations that are often complementary to those of homogenate binding assays. Performance of rapid kinetic association and dissociation assays and detailed multisite saturation or competition assays can be more difficult by autoradiography than with the use of tissue homogenates. This is due to temporal limitations of intact-tissue kinetics and to limits on the number of assays that can be performed on small structures represented in only a few tissue sections. Conversely, the very high sensitivity and spatial resolution of autoradiography affords the ability to study structures too small to accurately dissect for homogenate assays. The ability to combine binding-site autoradiography with other anatomical imaging procedures, including routine histological staining, histochemical staining, immunohistochemistry, nucleic acid hybridization assays, and some in vivo radioligand distribution assays, permits powerful and flexible multidisciplinary protocols not possible with homogen- ate assays. The ability to perform multiple assays in individual brains is an important asset in the study of brain pathology, where subjects may have variable severity and locations of abnormality. Autoradiographic screening of wide anatomic areas precludes the need to prospectively identify all areas of potential interest by gross dissection, as is necessary for homogenate tissue assays.

Pharmaceutical development may be assisted both by in vitro and in vivo ligand autoradiography. Although in vitro methods are preferred for the detailed characterization of binding-site pharmacology, in vivo distribution studies continue to be of use in determining the detailed biodistributions of new drugs, affording important data on pharmacokinetics and regional distribution in preclinical animal studies. By extension, emission tomographic imaging studies of drug distribution in the living human brain can provide important data on the distribution and effects of novel agents both in controls and disease states. This information may become increasingly important in the search for more effective drugs and in attempts to better distinguish therapeutic effects from unwanted side effects.

\section{RADIOLIGAND SELECTION}

\section{Isotopic Label}

Identification of candidate radioligands for autoradiography of binding sites first necessitates consideration of the possible radioisotopes for their labeling. The most widely employed isotope, tritium $\left({ }^{3} \mathrm{H}\right)$, has the advantages of low cost, ease of safe handling and disposal, and a wide variety of approaches for introducing label into ligands. Tritium is a neutron-rich hydrogen isotope derived from nuclear fission reactor bombardment. It decays by the conversion of a neutron to a proton with emission of a $\beta^{-}$particle and an antineutrino, thus increasing the number of protons in the nucleus and resulting in an atom of helium. The process of $\beta^{-}$emission results in a spectrum of emitted particle energies resulting from the differential distribution of the transition energy between the antineutrino and the $\beta^{-}$particle. The average $\beta^{-}$energy is approximately one-third of the maximum transition energy, and in the instance of ${ }^{3} \mathrm{H}$ is $5.7 \mathrm{keV}$. Tritium is unique among the isotopes employed for autoradiography since 
Table 1.4.1 Properties of Some Nuclides Useful in Autoradiography

\begin{tabular}{lcccccccc}
\hline Nuclide & Emission & $\begin{array}{c}\text { Max. } \\
\text { energy } \\
(\mathrm{keV})\end{array}$ & $\begin{array}{c}\text { Avg. } \\
\text { energy } \\
(\mathrm{keV})\end{array}$ & $\begin{array}{c}\text { Max. tissue } \\
\text { range }(\mathrm{mm})\end{array}$ & $\begin{array}{c}\text { Avg. tissue } \\
\text { range }(\mathrm{mm})\end{array}$ & $\begin{array}{c}\text { Half-life } \\
\begin{array}{c}\text { Max. sp. act. } \\
(\mathrm{Ci} / \mathrm{mol})\end{array}\end{array} \begin{array}{c}\text { Particles per } \\
\text { transition }\end{array}$ \\
\hline${ }^{3} \mathrm{H}$ & $\beta^{-}$ & 18.6 & 5.7 & 0.008 & 0.004 & 12.3 years & $2.9 \times 10^{4}$ & 1.00 \\
${ }^{14} \mathrm{C}$ & $\beta^{-}$ & 156 & 49 & 0.301 & 0.042 & 5370 years & $6.7 \times 10^{1}$ & 1.00 \\
${ }^{18} \mathrm{~F}$ & $\beta^{+}$ & 635 & 250 & 2.440 & 0.637 & $1.83 \mathrm{hr}$ & $1.7 \times 10^{9}$ & 1.00 \\
${ }^{32} \mathrm{P}$ & $\beta^{-}$ & 1710 & 695 & 8.210 & 2.750 & 14.3 days & $9.2 \times 10^{6}$ & 1.00 \\
${ }^{33} \mathrm{P}$ & $\beta^{-}$ & 250 & 77 & 0.637 & 0.092 & 25 days & $5.1 \times 10^{6}$ & 1.00 \\
${ }^{35} \mathrm{~S}$ & $\beta^{-}$ & 167 & 49 & 0.336 & 0.042 & 87 days & $1.5 \times 10^{6}$ & 1.00 \\
${ }^{99} \mathrm{~m}{ }^{12}$ & $\mathrm{ICE}^{a}$ & 120 & 120 & 0.195 & 0.195 & $6.01 \mathrm{hr}$ & $5.2 \times 10^{8}$ & 0.09 \\
${ }^{123} \mathrm{I}$ & $\mathrm{ICE}^{a}$ & 127 & 127 & 0.214 & 0.214 & $13.2 \mathrm{hr}$ & $2.4 \times 10^{8}$ & 0.14 \\
${ }^{125} \mathrm{I}$ & $\mathrm{AuE}^{131}$ & 30 & $24^{c}$ & 0.019 & 0.012 & 60 days & $2.2 \times 10^{6}$ & 0.20 \\
\hline
\end{tabular}

\footnotetext{
${ }^{a}$ Internal conversion electron decay.

${ }^{b}$ Auger electron decay.

${ }^{c}$ For ${ }^{125} \mathrm{I}$ (which exhibits Auger electron decay), the average energy is calculated from the abundance-weighted mean of electron energies resulting from ten distinct transition possibilities with energies ranging from 22 to $30 \mathrm{keV}$.
} Receptor Binding
Techniques its average $\beta^{-}$energy permits travel through only a few micrometers of tissue before its absorption (Table 1.4.1). Typical frozen histological tissue sections are much thicker than the average ${ }^{3} \mathrm{H} \beta^{-}$particle path length; sections of 15 to $20 \mu \mathrm{m}$ or greater are "infinitely thick" with respect to ${ }^{3} \mathrm{H}$ emissions. Autoradiograms of tritium-ligand distribution in most tissue sections are thus insensitive to minor inconsistencies in sectioning precision. The advantage afforded by this insensitivity to tissue-section thickness is counterbalanced by the effects of tissue self-absorption and by the need for specialized films and emulsions for detection of the low-energy ${ }^{3} \mathrm{H} \beta^{-}$emissions (see discussion of Contact Autoradiography).

As depicted in Table 1.4.1, radionuclides employed for autoradiography differ in mode of decay as well as in physical half-life, each of which affect the feasibility of their use in binding-site autoradiography. As a general guide, if binding sites are present at an average tissue concentration of 10 to $100 \mathrm{nM}$, a single tritium atom per ligand molecule will permit autoradiographic imaging with exposure times of 5 to 7 days and specialized tritium-sensitive $\mathrm{X}$-ray films. Increasing the number of labeled atoms per molecule or increasing the exposure time will each independently lower the detection threshold. If the known target binding-site density is substantially lower, use of a shorterhalf-life nuclide at higher specific activity per labeled atom may be necessary.
The most commonly employed nuclide with very high specific activity is iodine-125 ( $\left.{ }^{125} \mathrm{I}\right)$, obtainable at almost 100-fold higher activity per atom on the basis of its shorter half-life. However, additional features, including abundance of charged particulate emissions per decay, effective film-exposure index per particle, and the number of nuclide atoms per ligand molecule additionally influence the relative sensitivities of ligands labeled with ${ }^{125}$ I versus those labeled with ${ }^{3} \mathrm{H}$. Labeling of ligands with ${ }^{125}$ I may be accomplished by replacement of stable ${ }^{127}$ I or by iodination of a parent ligand at a position distant from its site of interaction with the binding site of interest. Iodine-bearing ligands are often more challenging to employ in vitro than their non-iodinated counterparts because of the effects of increased molecular size and greater lipophilic character conferred by iodine. These properties tend to slow the kinetics of ligand diffusion, binding, and dissociation-and also promote increased nonspecific tissue binding when assays employ polar, saline-based buffers. The radionuclide decay scheme of ${ }^{125} \mathrm{I}$ is more complicated than that of ${ }^{3} \mathrm{H}$. It decays by electron capture, a process involving absorption of an inner-shell electron (most often a K-shell electron) by the nucleus, which results in conversion of a proton to a neutron with emission of a neutrino. The orbital vacancy left by the captured electron is then filled by an electron from a higher energy shell, leaving the atom with excess energy. The 
atom then dissipates this energy by the emission of $\mathrm{X}$ rays or ejection of electrons. A given radionuclide will emit a mix of $X$ rays and electrons, depending on the energy to be dissipated and on the nuclear and electron composition of the atom. The $\mathrm{X}$ rays are termed "characteristic X rays." The emitted electrons, termed "Auger electrons," are the principal means of autoradiographic detection of ${ }^{125} \mathrm{I}$. The energy of the Auger electron is characteristic of the difference in energy states of the initial electron transition as well as the energy of the electron absorbing the transition energy. In the decay of ${ }^{125}$ I, Auger electrons of ten individual energies within the range of 20 to 30 $\mathrm{keV}$ are emitted with a cumulative probability of $20 \%$. Thus, only one in five decaying ${ }^{125}$ I atoms emit an Auger electron likely to interact with autoradiographic film (see discussion of Basic Autoradiographic Principles). The energies of these Auger emissions, however, are sufficiently higher than those of ${ }^{3} \mathrm{H} \beta^{-}$particles so that their tissue-penetration distances are similar to typical frozen-section thicknesses. Also, the effective film exposure per particle is much greater, and traditional anti-scratchcoated X ray film can be used for autoradiography. Overall, autoradiographic detection sensitivity per ${ }^{125}$ I-decay particle may be 5 - to 10 -fold higher than for ${ }^{3} \mathrm{H}$, and when this is combined with the differences in specific activity and particle yield per decay, autoradiographic detection may be enhanced by 50 - to 100 -fold over ${ }^{3} \mathrm{H}$ at the same tissue concentrations.

Other radionuclides have been employed successfully for autoradiography involving the emissions of $\beta^{-}$particles, including ${ }^{14} \mathrm{C},{ }^{32} \mathrm{P},{ }^{33} \mathrm{P}$, ${ }^{35} \mathrm{~S}$, and ${ }^{131} \mathrm{I}$. Of these nuclides, all but ${ }^{14} \mathrm{C}$ have specific activities adequate for routine autoradiographic imaging of low-abundance binding sites such as neurotransmitter receptors and transporters. The emitted energies of these tracers are all high enough to penetrate typical frozen-section tissue thicknesses well; furthermore these isotopes are not subject to tissue self-absorption concerns and do not require specialized films or emulsions for detection. However, the range of ligands that can be labeled with these nuclides is more limited than with ${ }^{3} \mathrm{H}$, based on their infrequent occurrence in active drugs.

The $\beta^{+}$emitter ${ }^{18} \mathrm{~F}$ has proven useful in positron emission tomographic (PET) imaging of neuroreceptors and other binding sites when incorporated in ligands to replace stable ${ }^{19} \mathrm{~F}$, when substituted for $\mathrm{OH}$ groups, or when in- corporated in an alkyl substituent (e.g., when employing fluoromethyl or fluoroethyl substitutions for methyl or ethyl groups). The process of $\beta^{+}$emission involves the conversion of a proton to a neutron with the emission of the $\beta^{+}$ particle and a neutrino from the nucleus. In the instance of ${ }^{18} \mathrm{~F}$, decay to stable ${ }^{18} \mathrm{O}$ involves emission of a $\beta^{+}$particle with maximum energy of $635 \mathrm{keV}$, but as in the instance of $\beta^{-}$decay, division of energy between the $\beta^{+}$particle and neutrino results in a spectrum of emitted $\beta^{+}$ energies averaging $\sim 250 \mathrm{keV}$. The emitted particles are effective in exposing autoradiographic film, although they are of such high energy that anatomic resolution is considerably less than that typically achieved with either ${ }^{3} \mathrm{H}$ or ${ }^{125} \mathrm{I}$. An additional consideration is the ultimate fate of the $\beta^{+}$particle, since after loss of its initial kinetic energy it undergoes annihilation with an electron in the absorbing medium whereby the $\beta^{+}$particle and electron are converted to $511-\mathrm{keV}$ photons. These electromagnetic radiations, indistinguishable in properties from $\gamma$ rays and $X$ rays, require the use of shielding in the laboratory to reduce exposure of personnel during in vitro assays. Although the short half-life of ${ }^{18} \mathrm{~F}$ can present challenges in tissue processing, this isotope has been successfully employed for ex vivo detection of ligand distributions. It would theoretically be possible to use ${ }^{18} \mathrm{~F}$ for in vitro assays as well if local cyclotron and chemistry facilities for radioligand syntheses are available.

The nuclides technetium- $99 \mathrm{~m}\left({ }^{99 \mathrm{~m}} \mathrm{Tc}\right)$ and iodine-123 ( $\left.{ }^{123} \mathrm{I}\right)$ can decay by emission of internal conversion electrons with probabilities of $9 \%$ and $14 \%$, respectively, per transition. These particles, like Auger electrons, are orbital electrons ejected from the atom after absorbing energy from a nuclear transition. In the instance of ${ }^{99 \mathrm{~m}} \mathrm{Tc}$, the transition is from an excited nuclear energy state (a metastable excited state denoted by the " $\mathrm{m}$ " in ${ }^{99 \mathrm{~m}} \mathrm{Tc}$ ) to a more stable nucleus of unchanged proton/neutron composition. In $\sim 89 \%$ of ${ }^{99 \mathrm{~m}} \mathrm{Tc}$ to ${ }^{99} \mathrm{Tc}$ transitions, energy is released as a $\gamma$ ray of $140 \mathrm{keV}$. In one of several alternative transition routes, however, energy is ultimately released as an internal conversion electron of $120 \mathrm{keV}$. In the case of ${ }^{123}$ I, initial transition by electron capture results in conversion of a proton to a neutron, and $\sim 14 \%$ of transitions lead subsequently to the emission of an internal conversion electron of $127 \mathrm{keV}$ that is suitable for autoradiographic imaging. Because ${ }^{99 \mathrm{~m} T c}$ is a transition metal, it can be incorporated by chelation or coordination chemistry into organic ligands; however,
Neuroanatomical Methods

\subsection{3}


as in the instance of iodination, the resulting ligands generally have much larger molecular sizes than related parent drugs. The most common autoradiographic application of ${ }^{99 \mathrm{~m}} \mathrm{Tc}$ is ex vivo imaging of cerebral perfusion on the basis of in vivo accumulation of the tracers [ $\left.{ }^{99 \mathrm{~m}} \mathrm{Tc}\right]$ hexamethylpropyleneamine oxime (HMPAO) or [ $\left.{ }^{99 \mathrm{~m}} \mathrm{Tc}\right] N, N^{\prime \prime}$-1,2-ethylene-dibisL-cysteine diethyl ester hydrochloride (ECD). Radionuclides with complicated transition schemes-e.g., ${ }^{99 \mathrm{~m}} \mathrm{Tc},{ }^{123} \mathrm{I},{ }^{125} \mathrm{I}$, and ${ }^{131} \mathrm{I}-\mathrm{most}$ often give rise to multiple forms of emitted energy, including $\gamma$ rays employed in medical diagnostic imaging. While these radionuclides are useful in tissue autoradiographic assays, this aspect necessitates consideration of additional shielding during in vitro assays, which is not necessary with the use of pure, low-energy $\beta^{-}$emitters such as ${ }^{3} \mathrm{H},{ }^{14} \mathrm{C},{ }^{33} \mathrm{P}$, and ${ }^{35} \mathrm{~S}$.

\section{Pharmacological and Chemical Ligand Profiles}

Ligands selected for in vitro autoradiographic assays share many characteristics with ligands employed for homogenate binding assays. The most useful ligands have high affinity for a well-defined population or populations of binding sites, exhibit relatively low nonsaturable or nonspecific binding, and have kinetic properties facilitating convenient equilibriumbinding assay development. Generally speaking, antagonist ligands that do not distinguish different receptor states on the basis of effector coupling provide the best tools for quantification of binding-site numbers. As in the instance of homogenate binding assays, ligand polarity may influence the ability to interact with the full tissue complement of binding sites. The use of highly polar ligands, such as quaternary amines, may limit detection to receptors exposed on membrane surfaces, and, in intact cells, to cell-surface receptors. Lipophilic ligands often detect additional binding sites presumed to reside in more lipophilic domains. In the example of binding to intact cells, these may include intracellular or internalized receptors. In homogenate assays and other broken-cell preparations, lipophilic ligands may still interact with a larger number of binding sites than polar ligands, suggesting that intramembranous receptor location or adjacent lipophilic membrane domains may be important. Ligand polarity/lipophilicity is most readily estimated on the basis of partitioning between aqueous and organic phases. A widely employed system uses octanol and saline phases. This system has been sufficiently studied to enable the estima- tion of the partitioning properties of new ligands by extrapolation from measurements made on related compounds (i.e., from the partitioning behavior of the parent molecular structure and its substituents). Ligands with octanol:saline partition coefficients less than unity are sufficiently polar that they do not cross cell membranes in intact cells and are likely to detect binding sites only in hydrophilic domains. Ligands with partition coefficients $>10$ are sufficiently lipophilic that they enter membranes readily, and are likely to detect binding sites in both lipophilic and hydrophilic domains. Ligands with partition coefficients $>1000$ pose additional problems in in vitro assays, which result from nonspecific binding. These ligands, encountered frequently among iodinated compounds, have very poor solubility in saline-based buffers and tend to distribute avidly into tissue sections in binding assays. The addition of carriers in the assay buffer (e.g., albumin) or reduction in buffer polarity by isoosmotic substitution of sucrose for sodium chloride may be necessary to reduce nonspecific binding in some instances.

\section{CHARACTERIZATION OF LIGAND-BINDING ASSAYS}

\section{General Aspects of Ligand-Binding Autoradiography}

Autoradiographic ligand assays share parallel aspects of experimental design with conventional in vitro homogenate binding assays, described comprehensively elsewhere (Yamamura et al., 1985, 1990). A tissue source must be selected and preprocessed, tissue samples are incubated in the presence of labeled ligand under carefully controlled conditions, the labeled tissue is washed to remove nonspecifically bound ligand, and specifically bound ligand is quantified. Although there are some important distinctions between autoradiographic and homogenate studies, the principles-reversible bimolecular binding processes as well as kinetic, saturation, and competition analyses-apply equally to both designs. In the following sections, general aspects and guidelines for performance of autoradiographic assays will be considered.

\section{Tissue Preparation}

Tissues suitable for homogenate binding assays are usually acceptable in autoradiographic analyses as well. In addition to specimens obtained at biopsy or necropsy of experimental animals, human surgical resection and post- 
mortem samples are often sufficiently preserved for autoradiography. In experimental animal studies, simple gross organ or tissue dissection followed by freezing is preferred. Perfusion fixation (UNIT 1.1), while sometimes affording improvements in histological, histochemical, or in situ nucleic acid hybridization studies, should generally be avoided in tissues that are to be used in ligand-binding experiments. Even very mild aldehyde or other tissue-fixation schemes may modify kinetic and pharmacological properties of binding sites. If fixed tissues must be employed, it is important to conduct all initial characterizations of the binding site(s) in similarly processed tissues. Effects of postmortem tissue handling and delay prior to dissection and freezing can theoretically influence the recovery of binding sites and other biochemical tissue markers. These considerations are particularly important in assays of human postmortem tissues, where intervals of many hours may pass before specimens are collected. Potential postmortem artifacts may be reduced by careful matching of sample histories between experimental groups. This approach, however, does not entirely guarantee that important information and distinctions have not been lost prior to tissue collection and storage. An additional approach to this problem is to conduct controlled experiments emulating human postmortem conditions and intervals in experimental animals, thereby permitting direct assessment of possible differences between immediate and delayed tissue dissection and freezing.

After dissection, freezing of small specimens can be readily accomplished by covering them in crushed dry ice. This method is applicable to tissue blocks of up to 3 to $5 \mathrm{~g}$ with good results. Blocks of considerably larger size require more efficient heat transfer during freezing to avoid microscopic ice-crystal formation or macroscopic fissuring. Large tissue blocks should be frozen by intermittent, repetitive immersion in isopentane chilled with dry ice (UNIT 1.1). Blocks should be dipped for several seconds at a time, followed by withdrawal for several seconds to permit equalization of temperature between the block surfaces and center. Otherwise, the surfaces will freeze first, and when the center subsequently freezes and expands the tissue may fracture. Frozen tissue blocks can be maintained for years if stored at $-70^{\circ} \mathrm{C}$ and protected from desiccation. Coating the surfaces of tissue blocks with a frozen-section embedding medium and packaging in airtight plastic freezer bags permits routine stor- age with good tissue preservation and maintenance of sectioning properties. Storage at higher temperatures or repeated temperature cycling from $-70^{\circ} \mathrm{C}$ to cryostat-sectioning temperature may hasten loss of some tissue biochemical reactivities. If there is concern that aging or repeated temperature cycling may alter or degrade binding-site properties, this can be explored directly in control tissues.

Tissue is prepared typically for in vitro autoradiographic binding assays by sectioning in a cryostat microtome (UNIT 1.1). Usual frozensection thicknesses of 10 to $30 \mu \mathrm{m}$ are acceptable for autoradiographic purposes. It is important to bear in mind when selecting tissue-sectioning conditions that generation of ${ }^{3} \mathrm{H}$ autoradiograms will not be appreciably affected by section thicknesses within this range, nor will section-to-section imprecision affect the autoradiograms. However, direct scintillation spectrometry assays of sections (see discussion of Determination of Incubation and Washing Parameters) and autoradiographic binding assays employing radionuclides with higher particulate-energy emissions will each be sensitive both to the selected tissue thickness and to the precision of sectioning. In the instance of ${ }^{3} \mathrm{H}$ autoradiography, it is typical to collect duplicate adjacent tissue sections to guard against the intrusion of artifacts in processing. With other radionuclides, triplicate sections should be considered, to reduce additional section-thickness error effects, unless the microtome precision is known to be excellent. Microtome precision can be assessed experimentally by comparison of autoradiographic densities of adjacent sections after incubation reactions with radionuclides of moderate to high penetration, or in ex vivo autoradiograms after the accumulation of tracers labeled with penetrating radionuclides. Motorized cryostat microtomes offer the benefit of improved section-thickness precision as compared with manual sectioning, but are considerably more costly than comparable manually driven models.

Tissue sections for use in autoradiographic binding assays are usually mounted frozen on microscope slides, thawed, and dried. Slides should be prewashed and subbed to promote section adhesion during binding assays. Subbing solutions employing gelatin (UNIT 1.1) are generally inferior to those containing poly-Llysine (see CPMB UNIT 14.1 and APPENDIX IA in this manual) for use in lengthy in vitro assays. High heat conditions should be avoided during section drying to prevent binding-site denatura-
Neuroanatomical Methods

\subsection{5}


tion. After complete drying, slide-mounted sections may generally be stored at $-70^{\circ} \mathrm{C}$ for up to several weeks without loss of binding activity. If a particular binding site is known to be fragile or is of unknown stability, effects of post-sectioning storage should be investigated directly in control experiments.

\section{Detection of Specific Binding}

Perhaps the most critical aspect of any ligand-binding assay is the detection and definition of saturable, specific binding. In the development of new assays, considerable attention should be focused on this aspect; when adopting assays from the published literature, it is equally important to review results of prior assay-development reports and give strong consideration to replicating critical methodological experiments prior to use of the assay.

Autoradiographic assays are comparable to homogenate binding experiments in the fundamentals of defining specific ligand binding. Specific binding is by definition saturable, thus, a minimum criterion is that the radioligand demonstrate a component of binding that does not increase linearly with free ligand concentration. This is most easily demonstrated by the effect of adding unlabeled ligand to the assay. More precise pharmacological characterization of specific binding is achievable when the radioligand and the unlabeled competitor are chemically dissimilar, minimizing the likelihood of multiple sites of overlapping tissue interaction. It is helpful to begin the search for specific binding of a new radioligand with knowledge of one or more unlabeled competitors of the ligand at the desired binding site as well as the inhibitory equilibrium binding constant $\left(K_{\mathrm{i}}\right)$ of each. It is best to employ an unlabeled competitor at concentrations not above $1000 \times K_{\mathrm{i}}$, resulting in occupancy of $99.9 \%$ of binding sites. Use of unnecessarily higher inhibitor concentrations increases the possibility of detecting unwanted, secondary sites rather than the binding site of interest. In the sections to follow, it is assumed that a definition of nonspecific radioligand binding has been formulated and that its basis is the use of a competitive inhibitor at an appropriate concentration relative to its $K_{\mathrm{i}}$, the radioligand concentration, and the radioligand's equilibrium binding constant $\left(K_{\mathrm{d}}\right)$.

\section{Determination of Incubation and Washing Parameters}

After establishing an initial definition of specific radioligand binding, it is important to determine critical assay parameters to maximize its separation from nonspecific binding. Preliminary studies to determine optimal incubation and post-incubation washing conditions are often performed without the use of autoradiography for radioligand measurement. In this instance, it is common to conduct binding studies identically to autoradiographic assays with the exception that after post-incubation washing, sections are wiped from the microscope slide with glass fiber filter paper and assayed by liquid scintillation counting or by $\gamma$ counting, depending on the radionuclide involved. In this way, rapid results can be obtained and multiple potential assay modifications and refinements tested in logical sequence. However, a series of tissue sections bearing reproducible numbers of binding sites is necessary for these assays. Even though section-thickness variation may have no effect on the autoradiographic detection of ${ }^{3} \mathrm{H}$, it is critical in these direct tissue assays.

As in the case of homogenate radioligandbinding assays, aspects of the basic binding incubation-including choice of buffer system, $\mathrm{pH}$, time, temperature, and duration of incubation-should be explored to maximize specific binding. Often, important initial estimates of these quantities can be derived from prior in vitro homogenate binding assays. It is generally advisable in autoradiographic assays, however, to employ incubation buffers that are isotonic, so as to avoid osmotic stresses on tissue sections. Hypotonic buffers are particularly problematic if tissue-section adhesion is marginal, and their use may result in tissue fragmentation and losses from the slide.

An advantage of autoradiographic binding assays over homogenate designs is the ability to sample the binding incubation buffer directly and analyze it during the course of the assay. In the early phases of assay development, direct measures of free ligand concentration and chromatography of the radioligand after tissue exposure can assist with detection of unanticipated technical artifacts. If ligand depletion resulting from metabolism is noted, a lower incubation temperature should be considered. If ligand depletion resulting from nonspecific binding is noted, alteration of the buffer properties to promote ligand solubility may help. If depletion is due to specific binding, the volume of incubation buffer per tissue section must be increased.

An initial parameter for assay optimization is the post-incubation washing time that best distinguishes specific from nonspecific bind- 
ing. Comparable tissue sections are usually incubated in solutions containing labeled radioligand with and without unlabeled competitor to block specific binding. Sections are then assayed for radioligand activity after varying post-incubation washing times, including an initial measurement without washing (i.e., a "zero wash time," or $t=0$ sample). These initial sections are processed by very brief ( 1 to $2 \mathrm{sec}$ ) dipping in buffer lacking both the ligand and competitor to remove binding assay buffer from the slide surface. This step is important for assessing the possibility of loss of labeled sites during prolonged washing. Varying wash times are assessed, comparing total, specific and nonspecific bindings to the initial $t=0$ estimates. Optimal post-incubation washing procedures should reduce nonspecific binding to a minimum, but not promote loss of any specific binding detected in $t=0$ samples (Frey and Howland, 1992). Prolonged washing procedures that enhance specific over nonspecific binding, but at the expense of lost specific binding, run the risk of preferentially revealing a subset of binding sites. Unless the nature of specific binding that remains versus that potentially lost in these processes is understood, experimental results may be biased and results of studies employing distinct radioligands targeting the same site(s) may be discordant.

Estimates of specific-to-nonspecific labeling of tissues derived from whole-section assays may actually provide minimum values in comparison with autoradiographic assays when ${ }^{3} \mathrm{H}$-labeled ligands are employed. This phenomenon is due to the likelihood that washing is most effective at the section surface, and most protracted at the section-slide interface where the diffusion distance to reach the buffer solution is greatest. Thus, the limited tissuedepth penetration of ${ }^{3} \mathrm{H} \beta^{-}$particles may result in autoradiographic images of the most favorable specific-to-nonspecific binding strata.

The appropriate tissue incubation time to achieve equilibrium in the radioligand assay should be determined prior to conducting saturation assays or other pharmacological characterizations. Since the forward ligand-receptor binding rate is a second-order process, it is sensitive to ligand and receptor concentration effects. The time required to achieve equilibrium is, therefore, a function of radioligand concentration, and will be longest at the lowest ligand concentration studied. Thus, it is useful to have initial estimates of radioligand $K_{\mathrm{d}}$ (see UNIT 7.5) so that equilibration time for ligand concentrations below the half-maximal bind- ing level can be safely used in saturation analyses. In practice, equilibration-time determinations are straightforward in design, involving incubations of tissue with radioligand for progressively increasing durations, followed by routine post-incubation washing and assay of bound tracer. A caveat, however, is the possible loss of intact binding sites in tissue sections during prolonged incubations. This may arise, in theory, because of solubilization of some sites into the binding buffer. Alternatively, losses of high-affinity ligand recognition sites may reflect proteolytic degradation or dissociation of multiple proteins or cofactors that are required together for ligand binding. If ligandbinding sites are lost over the interval of the assay, apparent equilibration may take place, but the data will actually reflect competition between increasing ligand binding and decreasing total binding capacity $\left(B_{\max }\right)$. This aspect of ligand equilibrium can be controlled by conducting parallel assays at the lowest ligand concentration of interest and at a higher, saturating concentration where binding equilibrates more rapidly. The low-concentration data are used to assess equilibration rate, while lack of decrease in binding over time at the high concentration will exclude the possibility of binding-site losses during incubation. Desirable assay characteristics include incubation times sufficiently short that no detectable losses of binding sites occur, but which readily permit equilibration of sub- $K_{\mathrm{d}}$ ligand concentrations. Ligands that do not readily equilibrate at low concentrations may, nevertheless, be useful for assays of regional $B_{\max }$ distribution alone. However, it is recommended that use of a nearsaturating ligand concentration for this purpose be used to guard against effects of possible unrecognized affinity differences that cannot be explored directly because of protracted equilibration times at low ligand concentration.

\section{Saturation Studies}

In addition to regional mapping of ligandbinding sites, assays to determine the pharmacological properties of saturable binding sites can be conducted by quantitative autoradiography (Penney et al., 1981). In particular, saturation binding assays are indispensible for evaluation of new radioligands in autoradiography. Although in vitro homogenate binding assays are often initial sources for selection of ligands for autoradiographic use, it is uncommon that kinetic association and dissociation binding rates or the equilibrium dissociation constant are identical in tissue homogenates and intact
Neuroanatomical Methods

\subsection{7}


tissue sections. Some differences may relate to a higher degree of compartmentalization of sites in intact sections, limiting ligand diffusion initially to a single dimension (perpendicular to the section face). The lack of vigorous tissue disruption in autoradiographic assays may additionally promote maintained association of effector coupling of other proteins with the binding sites of interest, modifying the conformation and characteristics of ligand binding.

Autoradiographic ligand-binding saturation analyses should be designed and conducted in parallel with in vitro homogenate assays. A range of ligand concentrations is employed in incubations of slides bearing a series of adjacent tissue sections. At each concentration, or at regular, less frequent intervals, assessment of nonspecific binding is made in additional sections. A 100-fold range of ligand concentrations centered on the $K_{\mathrm{d}}$ constitutes an ideal selection for estimation of both affinity and binding capacity. As detailed previously, it is important to verify technical aspects of the assay conditions so that artifacts do not result in erroneous saturation curves. It is important to verify by direct assay of the binding buffer that ligand depletion has not occurred during the incubation. It is also important that sufficient time be allowed to permit equilibration of ligand and binding sites. Each of these considerations is more important the lower the ligand concentrations used, and failure to properly design assays will result in an underestimation of binding. These effects result in curvilinear Rosenthall (Scatchard) plots, mimicking effects of cooperativity at interacting binding sites.

\section{Competition Assays}

Competition assays often require six or more distinct incubation conditions per binding site to assess binding affinity and capacity. In instances of multiple receptor subtypes that are distinguished by the competing ligand, as many as 15 to 20 parallel conditions may be necessary. Homogenate binding studies are adapted to these designs, permitting a large number of individual samples with highly similar tissue and binding-site compositions. In autoradiographic assays, however, anatomic gradients and variability from section to section make identification of truly identical tissue samples difficult. As a result, there is a methodological trade-off between the detail of the experimental design (number of replicate sections per condition and number of conditions) and the ability to select sufficient adjacent tissue sections bear- ing similar receptor types and numbers. Success of detailed multisite competition assays is enhanced if large, relatively invariant brain regions are selected (e.g., cerebral or cerebellar cortical regions, striatum, or major thalamic nuclei). In order to guard against unanticipated anatomic receptor gradients, repetition of the total binding condition (absence of competitor) should be performed at regular anatomic intervals throughout a series of sections. If the total binding does not change over the anatomic extent of the sections, differences may be safely ascribed to effects of the competitor. If subtle gradients in total binding are detected, it may be possible to interpolate between the control sections and use an adjusted $B_{\max }$ for each anatomic level.

\section{QUANTIFICATION OF LIGAND BINDING}

\section{Basic Autoradiographic Principles}

Quantitative receptor autoradiography is a logical extension of qualitative autoradiographic techniques developed in the 1950s and 1960s for localization of radioactive tracers in tissue specimens (see Rogers, 1979, for review). All these techniques are based on the apposition of a photographic film or emulsion to materials containing a radioactive source. The passage of charged particulate emissions such as electrons, $\beta^{-}$, and $\beta^{+}$particles through the silver halide crystals of photographic emulsions results in the conversion of silver ions to metallic silver. During development, these metallic silver ions catalyze the transformation of whole silver halide crystals into metallic silver, with consequent opacification of the emulsion. While electromagnetic radiation in the form of $\gamma$ rays, $X$ rays, or annihilation photons is also capable of exposing X-ray film, direct interactions between these radiations and photographic emulsions are much less efficient than those involving charged particulate emissions. Thus, little if any contribution to tissue autoradiography with the radionuclides discussed previously is attributable to $\gamma$ - or X-ray emissions.

Numerous factors influence the response of autoradiographic emulsions to a radioactive source. These include the type of ionizing radiation emitted by the source, the energy of the ionizing particles, the thickness of the specimen containing the radioactive source, the distribution of radioactivity within the specimen, the distance from the specimen to the emulsion, the thickness of the emulsion, and the density 
of silver halide crystals within the emulsion. In quantitative receptor autoradiography, many of these issues are not a major concern as long as experiments are performed in a standardized manner.

\section{Contact Autoradiography}

The ligand-binding site interaction and the radionuclide employed impose technical constraints in autoradiographic binding studies. The reversible binding of most radioligands means that exposure to aqueous solutions after completion of the binding assay may result in unwanted losses in both the amount and anatomic localization of bound ligand. This means that the traditional method for detection and high-resolution localization of labeled macromolecules (proteins labeled by amino acid incorporation or nucleic acids labeled by incorporation of labeled nucleotides)-i.e., dipping slide-mounted specimens directly in liquid photographic emulsions, may result in migration or loss of label in binding-site autoradiography. As a result, methods have been developed to allow dry contact between labeled tissue sections and autoradiographic emulsions (Roth and Stumpf, 1969). In the first technique, which is an extension of the traditional emulsion-dipping method, acid-washed coverslips may be coated with an appropriate nuclear emulsion. Coverslips are dried, affixed to microscope slides bearing the labeled tissue sample fixed at one end with contact cement, and then fastened with a removable clip at the opposite end, apposing the emulsion to the sections. After exposure, the clips are removed and the coverslips are gently raised from the tissue opposite the side of permanent attachment, to permit development and fixation of the emulsion.

The more popular and more readily quantifiable autoradiographic method is to appose tissue sections to an appropriate acetate-backed film. Using double-sided tape or contact cement, sections on microscope slides or coverslips are mounted on paper or cardboard and placed in a rigid, light-tight cassette. Film is placed immediately on top of the sections and the cassette sealed. The cassettes are stored in a cool, dry environment for an appropriate period, and then the film is removed and developed. In this design, technical aspects of autoradiogram generation are simplified; however, the structural registration of the image with corresponding tissue sections is less precise than with the emulsion-dipping or coverslipapposition techniques.
In film-contact autoradiography applications, the radionuclide used has a marked influence on the choice of film and subsequent data analysis. By far the most common radionuclide is the $\beta^{-}$emitter ${ }^{3} \mathrm{H}$, on the basis of its previously-discussed favorable properties (see discussion of Isotopic Label). The relatively low energy of its $\beta^{-}$emissions means that these emissions travel shorter distances both through tissue sections and within film emulsions, necessitating specialized autoradiographic techniques. Typical photographic and autoradiographic films are protected by a gelatinous "antiscratch" layer overlaying the silver halide matrix, which is designed to reduce mechanical damage as well as chemographic and contactinduced silver reductions. In the instance of ${ }^{3} \mathrm{H}$, these protective layers are too thick for $\beta^{-}$ particles to effectively penetrate them, and the resulting autoradiographic sensitivity is extremely low. Thus, specialized autoradiographic films lacking the protective antiscratch layer are necessary for contact autoradiography. These films are more costly than conventional X-ray or other photographic films useful in autoradiography of higher-energy emitters, and must be handled with great care to prevent mechanical damage and exposure of the emulsion. Additional consideration must be given to the possibility of direct chemical interactions between these emulsions and the section and slide, as is also possible with liquid-emulsion and dipped-coverslip autoradiography. While specialized tritium-sensitive films will certainly work with ${ }^{14} \mathrm{C},{ }^{35} \mathrm{~S}$, and ${ }^{32} \mathrm{P}$, use of such film in detection of the higher-energy emissions of these isotopes is unnecessary. Conventional X-ray films or finer-grained mammography films work well for these radioisotopes; the finer-grained emulsions afford optimal anatomic resolution with ${ }^{14} \mathrm{C}$ and ${ }^{35} \mathrm{~S}$, but at the expense of longer exposure times.

\section{Film Densitometry and Radioligand Quantification}

Data analysis by film densitometry has become straightforward through the availability of commercial computer-assisted densitometric systems. The relative ease of quantitative densitometry conceals some potential pitfalls in the analysis of autoradiographic data. It is important to recall that the relationship between incident-radiation exposure and optical density on developed film is not linear over an indefinite range of exposures. At higher levels of exposure, optical density reaches a ceiling (film saturation) beyond which it no longer reflects
Neuroanatomical Methods

1.4.9 
additional incident-radiation exposure. Even before saturation is reached, the relationship between exposure and film optical density deviates significantly from linearity, with reduced resolution of differences in incident-radiation intensity. It is important to prepare and analyze autoradiograms under conditions in which there is a strong relationship between incidentradiation exposure and developed-film optical density. This precaution necessitates careful attention to the duration of film exposure and uniformity of development conditions.

A related problem is that of radioactivity standards. To perform truly quantitative receptor autoradiography, tissue sections must be coexposed together with standards that provide an external reference for the relationship between radiation exposure and film optical density. Originally, investigators prepared their own standards by labeling brain paste homogenates (Freygang and Sokoloff, 1958; Pan et al., 1983) or plastics with known amounts of radioisotopes (Reivich et al., 1969; Pan et al., 1983). Plastic standards are now commercially available and widely used; they include examples of several radioisotopes used in receptor autoradiography. Standards containing shortlived radioisotopes must be replenished and recalibrated frequently. This is true even for ${ }^{3} \mathrm{H}$ standards if they are to be used over a period of years. Standards for ${ }^{14} \mathrm{C}$ have the advantage of not requiring recalibration because of the very slow rate of decay, and can be cross-calibrated against other radioisotopes to provide a universal set of standards. This concept, however, has been challenged by evidence that ${ }^{14} \mathrm{C}$ and ${ }^{3} \mathrm{H}$ plastic standards may not always produce parallel optical-density changes. In the authors' experience, this may occur when film reciprocity is violated in either of two ways. If the intensity of one, but not both, of the exposures has saturated, the exposures will no longer vary proportionately. In the instance of ${ }^{14} \mathrm{C}$ and ${ }^{3} \mathrm{H}$, most film emulsions permit ${ }^{14} \mathrm{C}$ access to deeper levels, resulting in higher maximal optical density and later saturation than with ${ }^{3} \mathrm{H}$. Additionally, if exposure times are very protracted (more than several months), fading of the latent images resulting from ${ }^{14} \mathrm{C}$ and ${ }^{3} \mathrm{H}$ may occur at distinct rates that are due to differing degrees of lattice distortion produced by deposition of high versus low $\beta^{-}$particle energies. Good correlations between relative ${ }^{14} \mathrm{C}$ and ${ }^{3} \mathrm{H}$ exposures are obtained if films are exposed for less than 3 months time and if care is taken to avoid saturation of the ${ }^{3} \mathrm{H}$ exposure. In addition,
${ }^{14} \mathrm{C}$ standards have been used to control for exposures arising from ${ }^{18} \mathrm{~F}$ (Olds et al., 1985), ${ }^{35} \mathrm{~S},{ }^{99 \mathrm{~m}} \mathrm{Tc}$ (Taylor et al., 1992), ${ }^{125} \mathrm{I}$, and ${ }^{123} \mathrm{I}$. On the basis of these experiences, it may be predicted that extension of this method of standardization to other nuclides listed in Table 1.4.1 should also succeed.

Another important consideration in the interpretation of $\left[{ }^{3} \mathrm{H}\right]$ ligand autoradiography is that differential absorption of emissions within tissue, known as tissue quenching, may alter imaging results. In brain tissue, lipid-rich white matter absorbs ${ }^{3} \mathrm{H}$ emissions to a greater extent than gray matter. This absorption differential gives rise to apparent ligand-concentration differences between brain regions that may in part reflect differing gray versus white-matter compositions. This effect may confer as much as a 2-fold difference in the autoradiographic image exposures of comparably labeled gray and white-matter structures (Geary and Wooten, 1985). Differential effects of ${ }^{3} \mathrm{H}$ quenching may be advantageous for anatomic localization of binding signals through contrast-enhancing effects. However, in between-group experimental comparisons, alterations in tissue composition rather than binding-site numbers may confound autoradiogaphic measures.

Three approaches have been employed to address the ${ }^{3} \mathrm{H}$ quenching problem. One design is to determine the relative changes in a reference binding site (a "control" site known to be experimentally unaffected) in comparison to the binding site of interest. If both sites apparently change by the same fraction between experimental groups, altered tissue composition is the most likely explanation. Some groups have developed procedures to eliminate lipids from dried tissue sections, thus, eliminating the major source of regional quenching differences (Happe and Murrin, 1990). Unfortunately, most delipidation procedures result in denaturation or loss of ligand-binding sites.

The final approach to evaluating and correcting for differential quenching effects is to make direct measures of attenuation of a diffusely introduced label in parallel sections to those in the binding assays. For example, terminal in vitro labeling of proteins followed by their autoradiography has been employed successfully for analysis and correction of tissue quenching effects (Smith et al., 1995). Quenching is not a significant source of bias or error in typical $20-\mu \mathrm{m}$-thick tissue sections when labeled with the higher-energy-emitting radionuclides.

\section{Receptor Binding Techniques}




\section{ANALYSES OF AUTORADIOGRAPHIC DATA}

\section{Anatomic Distribution}

Interpretation of autoradiograms requires the ability to identify reliably the correct regions of interest. Regional anatomy can be identified in several complementary ways. The most direct of these is use of the autoradiogram itself. With many ligands, there is sufficient detail and contrast in the images to establish regional boundaries, even for small regions. Fiber tracts typically express low levels of synaptic binding sites, and in the instance of tritium-labeled ligands, the higher lipid levels of fiber tracts will further reduce emulsion exposure (see discussion of quenching under Film Densitometry and Radioligand Quantitation). Consequently, structures such as the corpus callosum, the deep cerebellar white matter, the internal capsule, and and other myelinated fiber tracts are identified readily and may serve as anatomic landmarks for structural identification. Many binding sites also have regional gray-matter distribution patterns that permit ready identification of selected regions. For example, the striatal complex expresses uniquely high levels of several neurotransmitter receptors, including dopamine D1 and D2 receptors and adenosine A2 receptors, while cannabinoid receptors are expressed at very high levels within the globus pallidus (Herkenham et al., 1990). Autoradiograms of binding sites with more widespread patterns of expression-e.g., GABA (Pan et al., 1983), excitatory amino acid (Greenamyre et al., 1985), or muscarinic cholinergic receptors (Wamsley et al., 1980)—often contain sufficient regional variation in expression levels to permit accurate identification of regions.

Conventional histology can also be used to guide interpretation of autoradiograms. Sections exposed to film can be processed subsequently for Nissl staining (see discussion of Combination with Other Anatomic Methods) and then used for regional anatomic identification. Some image-processing systems (e.g., the MCID System family from Imaging Research; see SUPPLIERSAPPENDIX) support parallel processing and analysis of multiple images, allowing superposition of Nissl-stained sections and autoradiograms and direct transposition of structural boundaries from the histologic to the binding images. Sections immediately adjacent to those chosen for autoradiography can alternatively be used for histology. In addition to conventional histological tissue staining, sec- tions can be processed for histochemistry or other techniques that may reveal boundaries or tissue distinctions not appreciated with conventional histology.

\section{Saturation and Competition Isotherms}

Analyses of binding curves to estimate affinities and densities of sites are identical to the post-processing analyses conducted on data from in vitro homogenate binding studies. As mentioned previously, care should be taken to assure that radioligand $K_{\mathrm{d}}$ estimates used to make estimations of unlabeled ligand $K_{\mathrm{i}}$ values are appropriate to intact tissue sections (see discussion of Detection of Specific Binding). Ideally, all important binding parameters would be determined in each individual brain and region studied. This is often impractical, however, and $K_{\mathrm{d}}$ values determined in representative samples of an experimental group must often be extrapolated to regional mapping and unlabeled ligand competition experiments performed in other brains. Computer-assisted analyses (Munson and Rodbard, 1980) are of particular value when multiple binding-site estimations are undertaken to assure that unbiased parameter estimates are made and to permit proper statistical comparisons of alternative binding models. With typical autoradiographic measurement variances and difficulties in assuring stable anatomic receptor expression throughout a large series of adjacent tissue sections, it is unusual to identify and separate more than two sites in most binding experiments (Frey and Howland, 1992).

\section{Combination with Other Anatomic Methods}

Receptor autoradiography can be combined with almost any neuroanatomical method for which tissue preparation is compatible with receptor studies, using alternate sections derived from the same blocks of tissue for individual assays. Unfixed, frozen tissue sections can be used in a wide variety of neuroanatomic methods. In addition, some comparison measures can be made on the same tissue sections used for receptor autoradiography. Routine histology with Nissl staining is readily accomplished on sections used for receptor binding after film exposure, by post-fixing the sections over paraformaldehyde vapors. Sections are placed in a tightly sealed container in a rack or on a platform suspended above paraformaldehyde powder. The sections are exposed to paraformaldehyde vapors by warming of the chamber for a minimum of $48 \mathrm{hr}$, and then stained
Neuroanatomical Methods

1.4.11 
with $0.5 \%$ cresyl violet in the conventional manner (see UNIT 1.2). This method produces sections with excellent regional histology and surprisingly good preservation of perikaryal cellular morphology. The quality of these sections is more than adequate to establish regional boundaries and to confirm the presence of experimental lesions or cannula placements.

Another technique that can be readily combined with receptor autoradiography and that can employ either identical or alternate sections is $\left[{ }^{14} \mathrm{C}\right] 2$-deoxyglucose (2DG) autoradiography of cerebral glucose metabolism (Sokoloff et al., 1977). The same tissue sections utilized for 2DG studies can be processed for receptor autoradiography after the metabolic autoradiograms are initially obtained, or, more often, adjacent sections from $\left[{ }^{14} \mathrm{C}\right] 2 \mathrm{DG}$-labeled brains can be processed for ligand binding and autoradiograms exposed in parallel. The latter design is favored, since rapid desiccation of tissue sections on a slide warmer enhances the anatomic resolution of the 2DG method, but may diminish the recovery of binding sites. In any event, it is important to incorporate additional prewashes into the receptor autoradiography protocol to completely remove the $\left[{ }^{14} \mathrm{C}\right] 2 \mathrm{DG}$ from the tissue prior to binding assays with lower-energy radionuclides.

A number of additional techniques can be applied to alternate sections harvested during cryostat sectioning. Many histochemical techniques can be applied to fresh-frozen tissue sections. While somewhat lacking in cellular resolution, histochemistry on fresh-frozen sections can give valuable information about regional anatomy. Some investigators, for example, have used cytochrome oxidase histochemistry on fresh-frozen sections to estimate the extent of experimental lesions (Gonzalez and Garrosa, 1991). It is also possible to quantitate the intensity of many histochemical reaction products with densitometric methods analogous to those used for receptor autoradiograms. The absence of fixation eliminates a confounding variable for measurement of reaction-product density and can allow at least semiquantitative estimation of enzyme activity. Some investigators have developed methods to produce enzyme activity standards that can be processed in parallel with fresh-frozen tissue sections to yield a densitometric equivalent of using solution histochemistry to estimate enzyme activity (Biegon and Wolff, 1985).

Another useful neuroanatomical method that can be employed in fresh-frozen sections is in situ mRNA hybridization (see CPMB
Chapter 14 and UNIT 1.3 in this manual). Use of this technique together with receptor autoradiography on alternate sections allows complementary evaluations of regional mRNA and protein expressions; the ligand-binding assay provides the index of gene expression.

Tissue preparations for receptor autoradiography and immunohistochemistry are not generally compatible. Fixation necessary for the optimal visualization of many antigens often makes receptor autoradiography impossible. There are, however, some exceptions. Some proteins, such as the reactive astrocyte marker glial fibrillary acidic protein (GFAP; see UNIT 3.5), can be visualized by immunohistochemistry following alcohol or acetone post-fixation of fresh-frozen sections (also see UNIT 1.2). Further experimentation may expand the range of neuroanatomical methods compatible with receptor autoradiography.

\section{Three-Dimensional Reconstructions}

An emerging technique, developed initially for analysis of image data from positron emission tomography (PET) of blood flow in the human brain, involves the analysis of bindingsite distribution data throughout the entire brain volume at high resolution (Frey et al., 1996). This approach has been explored for its extension to experimental-animal autoradiographic imaging, with encouraging preliminary results after reconstructions of the entire brain from autoradiograms at regular intervals (Kim et al., 1994). Screening of the entire neuroaxis without the need for predefined regions of interest or preconceptions regarding regional boundaries may permit detection of unanticipated focal changes or of significant gradients within larger regions, which are not evident in qualitative examinations of autoradiograms. Conversely, these parallel analyses of large numbers of independent regions (voxels) require conservative statistical adjustments against false-positive results that may limit detection sensitivity. Experimental designs that employ limited numbers of a priori regional hypotheses, but which additionally include omnibus wholebrain survey techniques, may afford the greatest overall utility.

\section{CONCLUSION}

Ligand-binding autoradiography is a powerful technique with many strengths and advantages. Unlike most neuroanatomic techniques, it is readily quantifiable and provides numerical data that can be subjected to parametric statistical analyses. In addition to providing maps of 
binding-site distribution, it can also yield data about binding-site pharmacology and regulation that is often comparable to that obtained from homogenate binding techniques with less anatomical resolution. While receptor autoradiography does not typically provide cellularlevel resolution, it can provide excellent resolution on a brain-regional basis; with careful technique and selection of ligands it can distinguish and quantify small nuclear regions and subregions. Binding-site autoradiography is also very efficient with respect to numbers of experimental subjects needed in assays. Autoradiography often permits analyses of small brain structures that would require pooling of tissue from several subjects to obtain sufficient tissue for homogenate assays. Furthermore, it is technically easier to screen the entire neuroaxis in autoradiographic assays than to dissect and analyze a comparable number of discrete regions by homogenate binding methods. Tissue harvested for receptor autoradiography may also be used for routine histology, in situ mRNA hybridization, and histochemical techniques-allowing use of tissues for multiple complementary analyses.

Autoradiography provides unique information about the regional distribution of binding sites and may allow identification of otherwise unsuspected anatomical boundaries. The existence of striatal "patches" or "striosomes," for example, was documented first with $\mu$-opiate receptor autoradiography (Herkenham and Pert, 1981). Many other neuroanatomical boundaries and features are characterized by unique patterns of expression of receptors and other binding sites. The quantifiability of ligand-binding autoradiography also permits the documentation of more subtle changes such as subregional gradients of binding-site expression that may not be qualitatively obvious.

The association of specific populations of binding sites with specific populations of neurons or nerve terminals allows use of receptor autoradiography as a quantitative measure of the integrity of these neuronal elements. Ligand binding to the dopamine transporter or vesicular monoamine transporter, for example, have become standard techniques for assessment of the integrity of nigrostriatal dopaminergic terminals (Vander Borght et al., 1995). Receptor autoradiography is an established tool in examination of normal human neuroanatomy and in the characterization of neuropsychiatric pathologies. Benzodiazepine receptor autoradiography, for example, has been used to quantify neuronal losses in human temporal lobe epilepsy (Burdette et al., 1995). Receptor autoradiography in human postmortem tissue can be used to investigate the pharmacology of human binding sites, evaluate the fidelity of animal models of human disease states, and guide the selection of candidate probes for PET or single photon emission computed tomography (SPECT) studies of the living human brain.

Finally, the general method of receptor autoradiography can be applied to almost any ligand-binding-site interaction with suitable ligands and binding kinetics. Originally developed for analysis of neurotransmitter receptor populations, ligand-binding autoradiography is now employed routinely to study plasma membrane neurotransmitter reuptake transporters, synaptic vesicular neurotransmitter transporters, receptor-G-protein coupling, and biochemical second-messenger and ion-channel modulating effects of neurotransmitters, as well as enzyme expressions. The advantages of high anatomic resolution and robust quantifiability ensure that the autoradiographic approach will continue to provide valuable tools for future neuroscientific research.

\section{LITERATURE CITED}

Biegon, A. and Wolff, M. 1985. Quantitative histochemistry of acetylcholinesterase in rat and human brain postmortem. J. Neurosci. Methods 16:39-45.

Burdette, D.E., Sakurai, S.Y., Henry, T.R., Ross, D.A., Pennell, P.B., Frey, K.A., Sackellares, J.C., and Albin, R.L. 1995. Temporal lobe benzodiazepine binding in unilateral mesial temporal lobe epilepsy. Neurology 45:934-941.

Frey, K.A. and Howland, M.M. 1992. Quantitative autoradiography of muscarinic cholinergic receptor binding in the rat brain: Distinction of receptor subtypes in antagonist competition assays. J. Pharmacol. Exp. Ther. 263:1391-1400.

Frey, K.A., Minoshima, S., Koeppe, R.A., Kilbourn, M.R., Berger, K.L., and Kuhl, D.E. 1996. Stereotaxic summation analysis of human cerebral benzodiazepine binding maps. J. Cereb. Blood Flow Metab. 16:409-417.

Freygang, W.H. and Sokoloff, L. 1958. Quantitative measurement of regional circulation in the central nervous system by the use of radioactive inert gas. Adv. Biol. Med. Phys. 6:263-279.

Geary, W.A. and Wooten, G.F. 1985. Regional tritium quenching in quantitative autoradiography of the central nervous system. Brain Res. 336:334-336.

Gonzalez, L.F. and Garrosa, M. 1991. Quantitative histochemistry of cytochrome oxidase in rat brain. Neuroscience 123:251-253.

Greenamyre, J.T., Young, A.B., and Penney, J.B. 1985. Quantitative autoradiographic distribution
Neuroanatomical Methods

1.4.13 
of $\mathrm{L}-\left[{ }^{3} \mathrm{H}\right]$ glutamate binding sites in the rat central nervous system. J. Neurosci. 4:2133-2144.

Happe, H.K. and Murrin, L.C. 1990. Tritium quench in autoradiography during postnatal development of rat forebrain. Brain Res. 525:28-35.

Herkenham, M. and Pert, C.B. 1981. Mosaic distribution of opiate receptors, parafascicular projections and acetylcholinesterase in rat striatum. Nature 291:415-418.

Herkenham, M., Lynn, A.B., Little, M.D., Johnson, M.R., Melvin, L.S., De Kosta, B.R., and Rice, K.C. 1990. Cannabinoid receptor localization in brain. Proc. Natl. Acad. Sci. U.S.A. 87:19321936.

Kim, B., Frey, K.A., Mukhopadhyay, S., Ross, B.D., and Meyer, C.R. 1994. Three-dimensional registration of MRI and volumetric autoradiography. Radiology 193:363.

Munson, P.J. and Rodbard, D. 1980. Ligand: A versatile computerized approach for characterization of ligand-binding systems. Anal. Biochem. 107:220-239.

Olds, J.L., Frey, K.A., Ehrenkaufer, R.L., and Agranoff, B.W. 1985. A sequential double-label autoradiographic method that quantifies altered rates of regional glucose metabolism. Brain Res. 361:217-224.

Pan, H.S., Frey, K.A., Young, A.B., and Penney, J.B. Jr. 1983. Changes in $\left[{ }^{3} \mathrm{H}\right]$ muscimol binding in substantia nigra, entopeduncular nucleus, globus pallidus, and thalamus after striatal lesions as demonstrated by quantitative receptor autoradiography. J. Neurosci. 3:1189-1198.

Penney, J.B., Pan, H.S., Young, A.B., Frey, K.A., and Dauth, G.W. 1981. Quantitative autoradiography of $\left[{ }^{3} \mathrm{H}\right]$ muscimol binding in rat brain. Science 214:1036-1038.

Reivich, M., Jehle, J., Sokoloff, L., and Kety, S.S. 1969. Measurement of regional cerebral blood flow with antipyrine- $-{ }^{14} \mathrm{C}$ in awake cats. J. Appl. Physiol. 27:296-300.

Rogers, A.W. 1979. Techniques of Autoradiography. 3rd ed. Elsevier/North-Holland Biomedical Press, New York.

Roth, L.J. and Barlow, C.F. 1961. Drugs in the brain. Science 134:22-31.

Roth, L.J. and Stumpf, W.E. 1969. Autoradiography of Diffusible Substances. Academic Press, San Diego.

Smith, T.D., Gallagher, M., and Leslie, F.M. 1995. Cholinergic binding sites in rat brain: Analysis by age and cognitive status. Neurobiol. Aging 16:161-173

Sokoloff, L., Reivich, M., Kennedy, C., Des Rosiers, M.H., Patlak, C.S., Pettigrew, K.D., Sakurada, O., and Shinohara, M. 1977. The $\left[{ }^{14} \mathrm{C}\right]$ deoxyglucose method for the measurement of local cerebral glucose utilization: Theory, procedure and normal values in the conscious and anesthetized albino rat. J. Neurochem. 28:897-916.

Taylor, S.F., Frey, K.A., Baldwin, R.M., Papadopoulos, S.M., Petry, N.A., Rogers, W.L., McBride, B.J., Kerr, J.M., and Kuhl, D.E. 1992. Technetium-99m-N1-(2-mercapto-2-methylpropyl)N2-(2-propargylthio-2-methylpropyl)-1,2-ben zenediamine (T691): Preclinical studies of a potential new tracer of regional cerebral perfusion. J. Nucl. Med. 33:1836-1842.

Vander Borght, T.M., Sima, A.A.F., Kilbourn, M.R., Desmond, T.J., Kuhl, D.E., and Frey, K.A. 1995. $\left[{ }^{3} \mathrm{H}\right]$ Methoxytetrabenazine: A high specific activity ligand for estimating monoaminergic neuronal integrity. Neuroscience 68:955-962.

Wamsley, J.K., Zarbin, M.A., Birdsall, N.J.M., and Kuhar, M.J. 1980. Muscarinic cholinergic receptors: Autoradiographic localization of high and low affinity agonist binding sites. Brain Res. 200:1-12.

Yamamura, H.I., Kuhar, M.J., and Snyder, S.H. 1974. In vivo identification of muscarinic cholinergic receptor binding in rat brain. Brain Res. 80:170-176.

Yamamura, H.I., Enna, S.J., and Kuhar, M.J. 1985. Neurotransmitter Receptor Binding, 2nd ed. Raven Press, New York.

Yamamura, H.I., Enna, S.J., and Kuhar, M.J. 1990. Methods in Neurotransmitter Receptor Analysis. Raven Press, New York.

Young, W.S. III and Kuhar, M.J. 1979. A new method for receptor autoradiography: ${ }^{3} \mathrm{H}$-opioid receptor labeling in mounted tissue sections. Brain Res. 179:255-270.

Contributed by Kirk A. Frey

University of Michigan

Ann Arbor, Michigan

Roger L. Albin

University of Michigan and Ann Arbor Veterans' Administration Medical Center Ann Arbor, Michigan Receptor Binding
Techniques 